ESAIM: PROCEEDINGS AND SURVEYS, December 2020, Vol. 69, p. 70-78

Philippe Helluy, Jean-Marc Hérard, Nicolas Seguin

\title{
SOME MATHEMATICAL PROPERTIES OF A BAROTROPIC MULTIPHASE FLOW MODEL
}

\author{
Khaled Saleh ${ }^{1}$ and Nicolas Seguin ${ }^{2}$
}

\begin{abstract}
We study a model for compressible multiphase flows involving $N$ non miscible barotopic phases where $N$ is arbitrary. This model boils down to the barotropic Baer-Nunziato model when $N=2$. We prove the weak hyperbolicity property, the non-strict convexity of the natural mathematical entropy, and the existence of a symmetric form.
\end{abstract}

Keywords: Multiphase flows, Compressible flows, Hyperbolic PDEs, Entropy, Symmetrizable systems.

Mathematics Subject Classification: 76T30, 76T10, 35L60, 35Q35, 35F55.

\section{INTRODUCTION}

The modeling and numerical simulation of multiphase flows is a relevant approach for a detailed investigation of some patterns occurring in many industrial sectors.

In $4,8,9,14$, some modeling efforts have been provided for the design of compressible multiphase flow models allowing unique jump conditions and for which the initial-value problem is well posed. The $N$-phase flow models developed therein consist in an extension to $N \geq 3$ phases of the well-known Baer-Nunziato two phase flow model [1]. As in the Baer-Nunziato model, the PDEs are composed of a hyperbolic first order convective part consisting in $N$ Euler-like systems coupled through non-conservative terms and zero-th source terms accounting for pressure, velocity and temperature relaxation phenomena between the phases. It is worth noting that the latter models are quite similar to the classical two phase flow models in [2, 3,6 .

In [5, 11], two crucial properties have been proven for a class of two phase flow models containing the BaerNunziato model, namely, the convexity of the natural entropy associated with the system, and the existence of a symmetric form. As recalled in that paper, such properties are well understood for systems of conservation laws since Godunov 7 and Mock [13, but remain an open question for non conservative and non strictly hyperbolic models such as those considered here.

In the present paper, we prove the convexity of the entropy and the existence of a symmetric form for a barotropic multiphase flow model with $N$ - where $N$ is arbitrarily large - phases. We restrict the study to the case where the interfacial velocity coincides with one of the phasic material velocities.

\section{The Barotropic MUltiphase Flow MODEL}

We consider the following system of partial differential equations (PDEs) which is the convective part of the system introduced in 9 for the modeling of the evolution of $N$ distinct non-miscible compressible phases in a

${ }^{1}$ Université de Lyon, CNRS UMR 5208, Université Lyon 1, Institut Camille Jordan, 43 bd 11 novembre 1918; F-69622 Villeurbanne cedex, France.

${ }^{2}$ Irmar (UMR 6625), Université de Rennes 1, 263 avenue du Général Leclerc, CS 74205, 35042 RENNES Cedex, France.

(C) EDP Sciences, SMAI 2020

This is an Open Access article distributed under the terms of the Creative Commons Attribution License (http://creativecommons.org/licenses/by/4.0), which permits unrestricted use, distribution, and reproduction in any medium, provided the original work is properly cited.

Article published online by EDP Sciences and available at nttps://Www.esalm-proc.org or nittps://dol.org/10.1051/proc/202069070 
one dimensional space: for $k=1, . ., N, x \in \mathbb{R}$ and $t>0$ :

$$
\begin{aligned}
& \partial_{t} \alpha_{k}+u_{1} \partial_{x} \alpha_{k}=0, \\
& \partial_{t}\left(\alpha_{k} \rho_{k}\right)+\partial_{x}\left(\alpha_{k} \rho_{k} u_{k}\right)=0, \\
& \partial_{t}\left(\alpha_{k} \rho_{k} u_{k}\right)+\partial_{x}\left(\alpha_{k} \rho_{k} u_{k}^{2}+\alpha_{k} p_{k}\right)+\sum_{\substack{l=1 \\
l \neq k}}^{N} \mathscr{P}_{k l}(U) \partial_{x} \alpha_{l}=0 .
\end{aligned}
$$

The model consists in $N$ coupled Euler-type systems. The quantities $\alpha_{k}, \rho_{k}$ and $u_{k}$ represent the mean statistical fraction, the mean density and the mean velocity in phase $k$ (for $k=1, . ., N$ ). The quantity $p_{k}$ is the pressure in phase $k$. We assume barotropic pressure laws for each phase so that the pressure $p_{k}$ is a given function of the density $p_{k}: \rho_{k} \mapsto p_{k}\left(\rho_{k}\right)$ with the classical assumption that $p_{k}^{\prime}\left(\rho_{k}\right)>0$. The mean statistical fractions and the mean densities are positive and the following saturation constraint, which expresses the non-miscibility of the phases, holds everywhere at every time:

$$
\sum_{k=1}^{N} \alpha_{k}=1 .
$$

Thus, among the $N$ equations $1 \mathrm{a}$,,$N-1$ are independent and the main unknown $U$ is expected to belong to the physical space:

$$
\begin{aligned}
& \Omega_{U}=\left\{U=\left(\alpha_{2}, . ., \alpha_{N}, \alpha_{1} \rho_{1}, . ., \alpha_{N} \rho_{N}, \alpha_{1} \rho_{1} u_{1}, . ., \alpha_{N} \rho_{N} u_{N}\right)^{T} \in \mathbb{R}^{3 N-1},\right. \\
& \text { such that } \left.0<\alpha_{2}, . ., \alpha_{N}, \sum_{k=2}^{N} \alpha_{k}<1 \text { and } \alpha_{k} \rho_{k}>0 \text { for all } k=1, . ., N\right\} .
\end{aligned}
$$

Following [9], we make the following choice for the closure laws of the so-called interface pressures $\mathscr{P}_{k l}(U)$ :

$$
\begin{aligned}
& \text { for } k=1, \quad \mathscr{P}_{1 l}(U)=p_{l}\left(\rho_{l}\right), \quad \text { for } l=2, . ., N \\
& \text { for } k \neq 1, \quad \mathscr{P}_{k l}(U)=p_{k}\left(\rho_{k}\right), \quad \text { for } l=1, . ., N, l \neq k \text {. }
\end{aligned}
$$

Observing that the saturation constraint gives $\sum_{l=1, l \neq k}^{N} \partial_{x} \alpha_{l}=-\partial_{x} \alpha_{k}$ for all $k=1, . ., N$ the momentum equations (1c) can be simplified as follows:

$$
\begin{aligned}
& \partial_{t}\left(\alpha_{1} \rho_{1} u_{1}\right)+\partial_{x}\left(\alpha_{1} \rho_{1} u_{1}^{2}+\alpha_{1} p_{1}\left(\rho_{1}\right)\right)+\sum_{l=2}^{N} p_{l}\left(\rho_{l}\right) \partial_{x} \alpha_{l}=0, \\
& \partial_{t}\left(\alpha_{k} \rho_{k} u_{k}\right)+\partial_{x}\left(\alpha_{k} \rho_{k} u_{k}^{2}+\alpha_{k} p_{k}\left(\rho_{k}\right)\right)-p_{k}\left(\rho_{k}\right) \partial_{x} \alpha_{k}=0, \quad k=2, . ., N .
\end{aligned}
$$

\section{EigenstruCture of THE System}

The following result characterizes the wave structure of system (1):

Theorem 2.1. System (1) is weakly hyperbolic on $\Omega_{U}:$ it admits the following $3 N-1$ real eigenvalues: $\sigma_{1}(U)=. .=\sigma_{N-1}(U)=u_{1}, \sigma_{N-1+k}(U)=u_{k}-c_{k}\left(\rho_{k}\right)$ for $k=1, . ., N$ and $\sigma_{2 N-1+k}(U)=u_{k}+c_{k}\left(\rho_{k}\right)$ for $k=1, . ., N$, where $c_{k}\left(\rho_{k}\right)=\sqrt{p_{k}^{\prime}\left(\rho_{k}\right)}$. The corresponding right eigenvectors are linearly independent if, and only if,

$$
\left|u_{1}-u_{k}\right| \neq c_{k}\left(\rho_{k}\right), \quad \forall k=2, . ., N .
$$

The characteristic field associated with $\sigma_{1}(U), . ., \sigma_{N-1}(U)$ is linearly degenerate while the characteristic fields associated with $\sigma_{N-1+k}(U)$ and $\sigma_{2 N-1+k}(U)$ for $k=1, . ., N$ are genuinely non-linear. When (6) is violated, the system is said to be resonant. 
Proof. In the following, we denote $p_{k}$ and $c_{k}$ instead of $p_{k}\left(\rho_{k}\right)$ and $c_{k}\left(\rho_{k}\right)$ for $k=1, . . N$ in order to ease the notations. Choosing the variable $\mathcal{U}=\left(\alpha_{2}, . ., \alpha_{N}, u_{1}, p_{1}, . ., u_{N}, p_{N}\right)^{T}$, the smooth solutions of system (1) satisfy the following equivalent system:

$$
\partial_{t} \mathcal{U}+\mathscr{A}(\mathcal{U}) \partial_{x} \mathcal{U}=0
$$

where $\mathscr{A}(\mathcal{U})$ is the block matrix:

$$
\mathscr{A}(\mathcal{U})=\left(\begin{array}{c|ccc}
A & & \mathbf{0} & \\
\hline B_{1} & C_{1} & & \\
\vdots & & \ddots & \\
B_{N} & & & C_{N}
\end{array}\right)
$$

Defining $M_{k}=\left(u_{k}-u_{1}\right) / c_{k}$ the Mach number of phase $k$ relatively to phase 1 for $k=2, . ., N$, the matrices $A$, $B_{1}, . ., B_{N}$ and $C_{1}, . ., C_{N}$ are given as follows.

$$
\begin{aligned}
& A=\operatorname{diag}\left(u_{1}, . ., u_{1}\right) \in \mathbb{R}^{(N-1) \times(N-1)}, \\
& B_{1}=\left(\frac{1}{\alpha_{1} \rho_{1}} \sum_{k=2}^{N}\left(p_{k}-p_{1}\right) \delta_{i, 1} \delta_{j+1, k}\right) \underset{\substack{1 \leq i \leq 2 \\
1 \leq j \leq N-1}}{\in} \in \mathbb{R}^{2 \times(N-1)}, \\
& B_{k}=\left(\frac{\rho_{k} M_{k} c_{k}^{3}}{\alpha_{k}} \delta_{i, 2} \delta_{j+1, k}\right) \underset{\substack{1 \leq i \leq 2 \\
1 \leq j \leq N-1}}{ } \in \mathbb{R}^{2 \times(N-1)}, \quad \text { for } k=2, . ., N, \\
& C_{k}=\left(\begin{array}{cc}
u_{k} & 1 / \rho_{k} \\
\rho_{k} c_{k}^{2} & u_{k}
\end{array}\right), \quad \text { for } k=1, . ., N
\end{aligned}
$$

where $\delta_{p, q}$ is the Kronecker symbol: for $p, q \in \mathbb{N}, \delta_{p, q}=1$ if $p=q$ and $\delta_{p, q}=0$ otherwise. Since $A$ is diagonal and $C_{k}$ is $\mathbb{R}$-diagonalizable with eigenvalues $u_{k}-c_{k}$ and $u_{k}+c_{k}$, the matrix $\mathscr{A}(\mathcal{U})$ admits the eigenvalues $u_{1}$ (with multiplicity $N-1$ ),$u_{k}-c_{k}$ and $u_{k}+c_{k}$ for $k=1, . ., N$. In addition, $\mathscr{A}(\mathcal{U})$ is $\mathbb{R}$-diagonalizable provided that the corresponding right eigenvectors span $\mathbb{R}^{3 N-1}$. The right eigenvectors are the columns of the following block matrix:

$$
\mathcal{R}(\mathcal{U})=\left(\begin{array}{c|ccc}
A^{\prime} & & \mathbf{0} & \\
\hline B_{1}^{\prime} & C_{1}^{\prime} & & \\
\vdots & & \ddots & \\
B_{N}^{\prime} & & & C_{N}^{\prime}
\end{array}\right)
$$

where $A^{\prime}, B_{1}^{\prime}, . ., B_{N}^{\prime}$ and $C_{1}^{\prime}, . ., C_{N}^{\prime}$ are matrices defined by:

$$
\begin{aligned}
& A=\operatorname{diag}\left(1-M_{2}^{2}, . ., 1-M_{N}^{2}\right) \in \mathbb{R}^{(N-1) \times(N-1)} \\
& B_{1}^{\prime}=\left(-\frac{1}{\alpha_{1}} \sum_{k=2}^{N}\left(p_{k}-p_{1}\right)\left(1-M_{k}^{2}\right) \delta_{i, 2} \delta_{j+1, k}\right) \underset{\substack{1 \leq i \leq 2 \\
1 \leq j \leq N-1}}{1 \leq \mathbb{R}^{2 \times(N-1)},} \\
& B_{k}^{\prime}=\left(\left(-\frac{M_{k} c_{k}}{\alpha_{k}} \delta_{i, 1}+\frac{\rho_{k}\left(c_{k} M_{k}\right)^{2}}{\alpha_{k}} \delta_{i, 2}\right) \delta_{j+1, k}\right)_{\substack{1 \leq i \leq 2 \\
1 \leq j \leq N-1}} \in \mathbb{R}^{2 \times(N-1)},
\end{aligned}
$$

for $k=2, . ., N$,

$$
C_{k}^{\prime}=\left(\begin{array}{cc}
-1 & 1 \\
\rho_{k} c_{k} & \rho_{k} c_{k}
\end{array}\right), \quad \text { for } k=1, . ., N
$$

The first $N-1$ columns are the eigenvectors associated with the eigenvalue $u_{1}$. For $k=1, . ., N$, the $(N+2(k-1))$ th and $(N+(2 k-1))$-th columns are the eigenvectors associated with $u_{k}-c_{k}$ and $u_{k}+c_{k}$ respectively. We 
can see that $\mathcal{R}(\mathcal{U})$ is invertible if and only if $M_{k} \neq 1$ for all $k=2, . ., N$ i.e. if and only if inequations 6 hold. Denote $\left(\mathcal{R}_{j}(\mathcal{U})\right)_{1 \leq j \leq 3 N-1}$ the columns of $\mathcal{R}(\mathcal{U})$. If $1 \leq j \leq N-1$, we can see that the $N$-th component of $\mathcal{R}_{j}(\mathcal{U})$ is zero. This implies that for all $1 \leq j \leq N-1, \mathcal{R}_{j}(\mathcal{U}) \cdot \nabla_{\mathcal{U}}\left(u_{1}\right)=0$. Hence, the field associated with the eigenvalue $u_{1}$ is linearly degenerated. Now we observe that all the acoustic fields are genuinely non linear since for all $k=1, \ldots, N$ :

$$
\begin{gathered}
\mathcal{R}_{N+2(k-1)}(\mathcal{U}) \cdot \nabla_{\mathcal{U}}\left(u_{k}-c_{k}\right)=-1-\rho_{k} c_{k} \frac{\partial c_{k}}{\partial p_{k}} \neq 0 \\
\mathcal{R}_{N+(2 k-1)}(\mathcal{U}) \cdot \nabla_{\mathcal{U}}\left(u_{k}+c_{k}\right)=1+\rho_{k} c_{k} \frac{\partial c_{k}}{\partial p_{k}} \neq 0 .
\end{gathered}
$$

Proposition 2.2. The linearly degenerated field $\sigma_{1}(U)=. .=\sigma_{N-1}(U)=u_{1}$ admits the following $2 N$ independent Riemann invariants:

$$
\begin{aligned}
& \psi_{1}(U)=u_{1}, \\
& \psi_{2}(U)=\sum_{l=1}^{N}\left(\alpha_{l} p_{l}\left(\rho_{l}\right)+\alpha_{l} \rho_{l}\left(u_{l}-u_{1}\right)^{2}\right), \\
& \psi_{1+k}(U)=\alpha_{k} \rho_{k}\left(u_{k}-u_{1}\right), \quad \text { for } k=2, . ., N, \\
& \psi_{N+k}(U)=e_{k}\left(\rho_{k}\right)+\frac{p_{k}\left(\rho_{k}\right)}{\rho_{k}}+\frac{1}{2}\left(u_{k}-u_{1}\right)^{2}, \quad \text { for } k=2, . ., N .
\end{aligned}
$$

Proof. Denoting $\mathcal{U}=\left(\alpha_{2}, . ., \alpha_{N}, u_{1}, p_{1}, . ., u_{N}, p_{N}\right)^{T}$, one must check that for $p=1, . ., 2 N, \nabla_{\mathcal{U}} \psi_{p}(\mathcal{U}) \cdot \mathcal{R}_{j}(\mathcal{U})=0$ for all $j=1, . ., N-1$ where $\left(\mathcal{R}_{j}(\mathcal{U})\right)_{1 \leq j \leq N-1}$ are the eigenvectors associated with the eigenvalue $\sigma_{1}(\mathcal{U})=. .=$ $\sigma_{N-1}(\mathcal{U})=u_{1}$. The computation is tedious but straightforward.

\section{Mathematical Entropy}

An important consequence of the closure law (3) for the interface pressures $\mathscr{P}_{k l}(U)$ is the existence of an additional conservation law for the smooth solutions of (1). Defining the specific internal energy of phase $k, e_{k}$ by $e_{k}^{\prime}\left(\rho_{k}\right)=p_{k}\left(\rho_{k}\right) / \rho_{k}^{2}$ and the specific total energy of phase $k$ by $E_{k}=u_{k}^{2} / 2+e_{k}\left(\rho_{k}\right)$, the smooth solutions of (1) satisfy the following identities:

$$
\begin{aligned}
& \partial_{t}\left(\alpha_{1} \rho_{1} E_{1}\right)+\partial_{x}\left(\alpha_{1} \rho_{1} E_{1} u_{1}+\alpha_{1} p_{1}\left(\rho_{1}\right) u_{1}\right)+u_{1} \sum_{l=2}^{N} p_{l}\left(\rho_{l}\right) \partial_{x} \alpha_{l}=0, \\
& \partial_{t}\left(\alpha_{k} \rho_{k} E_{k}\right)+\partial_{x}\left(\alpha_{k} \rho_{k} E_{k} u_{k}+\alpha_{k} p_{k}\left(\rho_{k}\right) u_{k}\right)-u_{1} p_{k}\left(\rho_{k}\right) \partial_{x} \alpha_{k}=0, \quad k=2, . ., N .
\end{aligned}
$$

Summing for $k=1, . ., N$, the smooth solutions of (1) are seen to satisfy the following additional conversation equation which expresses the conservation of the total mixture energy :

$$
\partial_{t}\left(\sum_{k=1}^{N} \alpha_{k} \rho_{k} E_{k}\right)+\partial_{x}\left(\sum_{k=1}^{N}\left(\alpha_{k} \rho_{k} E_{k} u_{k}+\alpha_{k} p_{k}\left(\rho_{k}\right) u_{k}\right)\right)=0 .
$$

As regards the non-smooth weak solutions of (1), one has to add a so-called entropy criterion in order to select the relevant physical solutions. For this purpose, we prove the following result.

Theorem 3.1. For all $k=1, . ., N$, the fractional specific energy of phase $k$ defined by

$$
\left(\alpha_{k} \rho_{k} E_{k}\right): U \mapsto\left(\alpha_{k} \rho_{k} E_{k}\right)(U),
$$

is a non strictly convex function of $U$. Consequently, the total mixture energy, defined by $\left(\sum_{k=1}^{N} \alpha_{k} \rho_{k} E_{k}\right)(U)$ is also a non strictly convex function of $U$. In the light of $(10)$, the total mixture energy is a mathematical entropy of system (1). 
Proof. For all $k=1, . ., N$, define $V_{k}=\left(\rho_{k}, \rho_{k} u_{k}\right)^{T}$ the monophasic state vector of phase $k$ and define $U_{k}=$ $\left(\alpha_{k}, \alpha_{k} \rho_{k}, \alpha_{k} \rho_{k} u_{k}\right)^{T}=\left(\alpha_{k}, \alpha_{k} V_{k}^{T}\right)^{T}$. The monophasic mathematical entropy of phase $k$ is given by:

$$
\mathcal{S}_{k}\left(\rho_{k}, \rho_{k} u_{k}\right)=\mathcal{S}_{k}\left(V_{k}\right)=\rho_{k}\left(\frac{\left(\rho_{k} u_{k}\right)^{2}}{2 \rho_{k}^{2}}+e_{k}\left(\rho_{k}\right)\right) .
$$

Defining $\mathscr{S}_{k}\left(U_{k}\right)=\alpha_{k} \mathcal{S}_{k}\left(\frac{\alpha_{k} V_{k}}{\alpha_{k}}\right)$, we have $\left(\alpha_{k} \rho_{k} E_{k}\right)(U)=\mathscr{S}_{k}\left(U_{k}\right)$ for $k=1, . ., N$. Without loss of generality, we can rearrange the components of $U$ and assume that: $U=\left(\alpha_{1} \rho_{1}, \alpha_{1} \rho_{1} u_{1}, U_{2}^{T}, U_{3}^{T}, . ., U_{N}^{T}\right)^{T}$. Thus, for $k=2, . ., N,\left(\alpha_{k} \rho_{k} E_{k}\right)(U)$ solely depends on $U_{k}$ while $\left(\alpha_{1} \rho_{1} E_{1}\right)(U)$ depends on $\left(\alpha_{1} \rho_{1}, \alpha_{1} \rho_{1} u_{1}\right)$ and on all $U_{k}$ for $k=2, . ., N$ through its dependence on $\alpha_{1}=1-\sum_{k=2}^{N} \alpha_{k}$.

Case 1: Convexity of $\left(\alpha_{k} \rho_{k} E_{k}\right)(U)$ for $k=2, . ., N$ : The matrix $\left(\alpha_{k} \rho_{k} E_{k}\right)^{\prime \prime}(U)$ has the following block-diagonal structure for $k=2, . ., N$ :

$$
\left(\alpha_{k} \rho_{k} E_{k}\right)^{\prime \prime}(U)=\text { block-diag }\left(0_{\mathbb{R}^{2 \times 2}}, 0_{\mathbb{R}^{3 \times 3}}, . ., 0_{\mathbb{R}^{3 \times 3}}, \mathscr{S}_{k}^{\prime \prime}\left(U_{k}\right), 0_{\mathbb{R}^{3 \times 3}}, . ., 0_{\mathbb{R}^{3 \times 3}}\right) .
$$

Hence, $\left(\alpha_{k} \rho_{k} E_{k}\right)^{\prime \prime}(U)$ is a positive matrix if and only if $\mathscr{S}_{k}^{\prime \prime}\left(U_{k}\right)$ is a positive matrix. Since $\mathscr{S}_{k}\left(U_{k}\right)=$ $\alpha_{k} \mathcal{S}_{k}\left(\frac{\alpha_{k} V_{k}}{\alpha_{k}}\right)$, differentiating twice, we obtain that the matrix $\mathscr{S}_{k}^{\prime \prime}\left(U_{k}\right)$ is the $3 \times 3$ matrix given by:

$$
\mathscr{S}_{k}^{\prime \prime}\left(U_{k}\right)=\left(\begin{array}{c|c}
A_{k} & B_{k}^{T} \\
\hline B_{k} & C_{k}
\end{array}\right)
$$

with

$$
\begin{aligned}
A_{k} & =\frac{1}{\alpha_{k}} V_{k}^{T} \mathcal{S}_{k}^{\prime \prime}\left(V_{k}\right) V_{k} \in \mathbb{R}, \\
B_{k} & =-\frac{1}{\alpha_{k}} \mathcal{S}_{k}^{\prime \prime}\left(V_{k}\right) V_{k} \in \mathbb{R}^{2 \times 1}, \\
C_{k} & =\frac{1}{\alpha_{k}} \mathcal{S}_{k}^{\prime \prime}\left(V_{k}\right) \in \mathbb{R}^{2 \times 2} .
\end{aligned}
$$

Let be given $\left(a, \mathbf{b}^{T}\right)^{T} \in \mathbb{R}^{3 \times 1}$ with $a \in \mathbb{R}$ and $\mathbf{b} \in \mathbb{R}^{2 \times 1}$. Then, we easily see that:

$$
\begin{aligned}
\left(a, \mathbf{b}^{T}\right) & \mathscr{S}_{k}^{\prime \prime}\left(U_{k}\right)\left(a, \mathbf{b}^{T}\right)^{T} \\
& =a^{2} A_{k}+2 a B_{k}^{T} \mathbf{b}+\mathbf{b}^{T} C_{k} \mathbf{b} \\
& =a^{2} \frac{1}{\alpha_{k}} V_{k}^{T} \mathcal{S}_{k}^{\prime \prime}\left(V_{k}\right) V_{k}-2 a \frac{1}{\alpha_{k}} \mathbf{b}^{T} \mathcal{S}_{k}^{\prime \prime}\left(V_{k}\right) V_{k}+\frac{1}{\alpha_{k}} \mathbf{b}^{T} \mathcal{S}_{k}^{\prime \prime}\left(V_{k}\right) \mathbf{b} \\
& =\frac{1}{\alpha_{k}}\left(a V_{k}-\mathbf{b}\right)^{T} \mathcal{S}_{k}^{\prime \prime}\left(V_{k}\right)\left(a V_{k}-\mathbf{b}\right) .
\end{aligned}
$$

Since $\mathcal{S}_{k}^{\prime \prime}\left(V_{k}\right)$ is a positive matrix by the strict convexity of the monophasic mathematical entropy $\mathcal{S}_{k}$, the right hand side is positive, which yields the positivity of the matrix $\mathscr{S}_{k}^{\prime \prime}\left(U_{k}\right)$ and hence the (non-strict) convexity of $\left(\alpha_{k} \rho_{k} E_{k}\right)(U)$ for $k=2, . ., N$.

Case 2: Convexity of $\left(\alpha_{1} \rho_{1} E_{1}\right)(U)$ : We have

$$
\left(\alpha_{1} \rho_{1} E_{1}\right)(U)=\left(1-\sum_{k=2}^{N} \alpha_{k}\right) \mathcal{S}_{1}\left(\frac{\alpha_{1} V_{1}}{1-\sum_{k=2}^{N} \alpha_{k}}\right) .
$$


Thus, the Hessian matrix $\left(\alpha_{1} \rho_{1} E_{1}\right)^{\prime \prime}(U)$ has the following structure:

$$
\left(\alpha_{1} \rho_{1} E_{1}\right)^{\prime \prime}(U)=\left(\begin{array}{c|ccc}
C_{1} & -\mathbf{B}_{\mathbf{1}} & \cdots & -\mathbf{B}_{\mathbf{1}} \\
\hline-\mathbf{B}_{\mathbf{1}}^{\mathbf{T}} & \mathbf{A}_{\mathbf{1}} & \cdots & \mathbf{A}_{\mathbf{1}} \\
\vdots & \vdots & & \vdots \\
-\mathbf{B}_{\mathbf{1}}^{\mathbf{T}} & \mathbf{A}_{\mathbf{1}} & \cdots & \mathbf{A}_{\mathbf{1}}
\end{array}\right) .
$$

Defining $A_{1}, B_{1}$ and $C_{1}$ as in (11), the matrices $\mathbf{A}_{\mathbf{1}}$ and $\mathbf{B}_{\mathbf{1}}$ are given by:

$$
\mathbf{A}_{1}=\left(\begin{array}{ccc}
A_{1} & 0 & 0 \\
0 & 0 & 0 \\
0 & 0 & 0
\end{array}\right) \in \mathbb{R}^{3 \times 3}, \quad-\mathbf{B}_{1}=\left(\begin{array}{l|ll}
-B_{1} & 0 & 0 \\
0 & 0
\end{array}\right) \in \mathbb{R}^{2 \times 3} .
$$

Let be given $\mathbf{x}=\left(\mathbf{b}_{1}^{T}, a_{2}, \mathbf{b}_{2}^{T}, a_{3}, \mathbf{b}_{3}^{T}, . ., a_{N}, \mathbf{b}_{N}^{T}\right)^{T} \in \mathbb{R}^{(3 N-1) \times 1}$ with $a_{k} \in \mathbb{R}$ for $k=2, . ., N$ and $\mathbf{b}_{k} \in \mathbb{R}^{2 \times 1}$ for all $k=1, . ., N$. An easy computation gives:

$$
\mathbf{x}^{T}\left(\alpha_{1} \rho_{1} E_{1}\right)^{\prime \prime}(U) \mathbf{x}=\mathbf{b}_{1}^{T} C_{1} \mathbf{b}_{1}+\sum_{p=2}^{N}\left(\left(a_{p}, \mathbf{b}_{p}^{T}\right)\left(-\mathbf{B}_{\mathbf{1}}^{T} \mathbf{b}_{1}\right)+\sum_{k=2}^{N}\left(a_{p}, \mathbf{b}_{p}^{T}\right) \mathbf{A}_{\mathbf{1}}\left(a_{k}, \mathbf{b}_{k}^{T}\right)^{T}\right) .
$$

We easily check that

$$
\begin{aligned}
& \left(a_{p}, \mathbf{b}_{p}^{T}\right)\left(-\mathbf{B}_{\mathbf{1}}^{T} \mathbf{b}_{1}\right)=\left(a_{p}, \mathbf{b}_{p}^{T}\right)\left(-\mathbf{b}_{1}^{T} B_{1}, 0,0\right)^{T}=a_{p} \mathbf{b}_{1}^{T}\left(-B_{1}\right) \\
& \left(a_{p}, \mathbf{b}_{p}^{T}\right) \mathbf{A}_{\mathbf{1}}\left(a_{k}, \mathbf{b}_{k}^{T}\right)^{T}=\left(a_{p}, \mathbf{b}_{p}^{T}\right)\left(a_{k} A_{1}, 0,0\right)^{T}=a_{p} a_{k} A_{1} .
\end{aligned}
$$

Hence,

$$
\begin{aligned}
\mathbf{x}^{T}\left(\alpha_{1} \rho_{1} E_{1}\right)^{\prime \prime}(U) \mathbf{x} & =\mathbf{b}_{1}^{T} C_{1} \mathbf{b}_{1}+\sum_{p=2}^{N} a_{p} \mathbf{b}_{1}^{T}\left(-B_{1}\right)+\sum_{p=2}^{N} \sum_{k=2}^{N} a_{p} a_{k} A_{1} \\
& =\frac{1}{\alpha_{1}} \mathbf{b}_{1}^{T} \mathcal{S}_{1}^{\prime \prime}\left(V_{1}\right) \mathbf{b}_{1}+\frac{1}{\alpha_{1}}\left(\sum_{p=2}^{N} a_{p}\right) \mathbf{b}_{1}^{T} \mathcal{S}_{1}^{\prime \prime}\left(V_{1}\right) V_{1} \\
& +\frac{1}{\alpha_{1}}\left(\sum_{p=2}^{N} a_{p}\right) V_{1}^{T} \mathcal{S}_{1}^{\prime \prime}\left(V_{1}\right)\left(\sum_{k=2}^{N} a_{k}\right) V_{1} \\
& =\frac{1}{\alpha_{1}}\left(\left(\sum_{k=2}^{N} a_{k}\right) V_{1}+\mathbf{b}_{1}\right)^{T} \mathcal{S}_{1}^{\prime \prime}\left(V_{1}\right)\left(\left(\sum_{k=2}^{N} a_{k}\right) V_{1}+\mathbf{b}_{1}\right) .
\end{aligned}
$$

Since $\mathcal{S}_{1}^{\prime \prime}\left(V_{1}\right)$ is a positive matrix by the strict convexity of the monophasic mathematical entropy $\mathcal{S}_{1}$, the right hand side is positive, which yields the positivity of the matrix $\left(\alpha_{1} \rho_{1} E_{1}\right)^{\prime \prime}(U)$. Since $\mathbf{x}^{T}\left(\alpha_{1} \rho_{1} E_{1}\right)^{\prime \prime}(U) \mathbf{x}$ does not depend on $\mathbf{b}_{k}$ for $k=2, . ., N,\left(\alpha_{1} \rho_{1} E_{1}\right)^{\prime \prime}(U)$ is not positive definite and $\left(\alpha_{1} \rho_{1} E_{1}\right)(U)$ is non strictly convex.

The convexity of the total mixture energy is a direct consequence of the convexity of all the fractional specific energies and we have:

$$
\mathbf{x}^{T}\left(\sum_{k=1}^{N} \alpha_{k} \rho_{k} E_{k}\right)^{\prime \prime}(U) \mathbf{x}=0 \Longleftrightarrow \mathbf{x}=\left(-\left(\sum_{k=2}^{N} a_{k}\right) V_{1}^{T}, a_{2}, a_{2} V_{2}^{T}, . ., a_{N}, a_{N} V_{N}^{T}\right)^{T} \text { with }\left(a_{2}, . ., a_{N}\right) \in \mathbb{R}^{N-1} .
$$

Thus, the total mixture energy in non strictly convex. 


\section{Symmetrizability}

Definition 4.1. The system (1) is said to be symmetrizable if there exists a $C^{1}$-diffeomorphism $\mathbb{R}^{3 N-1} \rightarrow$ $\mathbb{R}^{3 N-1}, U \mapsto \mathcal{U}$, a symmetric positive definite matrix $\mathcal{P}(\mathcal{U})$, and a symmetric matrix $\mathcal{Q}(\mathcal{U})$ such that the smooth solutions of (1) satisfy:

$$
\mathcal{P}(\mathcal{U}) \partial_{t} \mathcal{U}+\mathcal{Q}(\mathcal{U}) \partial_{x} \mathcal{U}=0
$$

Since system (1) admits no conservative form and since the total mixture energy defined in the previous section is not strictly convex, we cannot use it to prove the symmetrizability of the system by multiplication by its hessian matrix. However we can find a suitable positive definite matrix $\mathcal{P}(\mathcal{U})$ which symmetrizes the system.

Theorem 4.1. System (1) is symmetrizable as long as the non resonance condition (6) holds.

Proof. Let us define $\mathcal{U}=\left(\alpha_{2}, . ., \alpha_{N}, u_{1}, p_{1}, . ., u_{N}, p_{N}\right)^{T}$. The smooth solutions of system (1) satisfy

$$
\partial_{t} \mathcal{U}+\mathscr{A}(\mathcal{U}) \partial_{x} \mathcal{U}=0
$$

where the matrix $\mathscr{A}(\mathcal{U})$ is given in $(7)$. Let us seek for a symmetric positive definite matrix $\mathcal{P}(\mathcal{U})$ that symmetrizes the system. We seek for $\mathcal{P}(\mathcal{U})$ in the form:

$$
\mathcal{P}(\mathcal{U})=\left(\begin{array}{c|ccc}
\theta \mathbb{I}_{N-1} & D_{1}^{T} & \ldots & D_{N}^{T} \\
\hline D_{1} & P_{1} & & \\
\vdots & & \ddots & \\
D_{N} & & & P_{N}
\end{array}\right), \quad \text { with } \quad P_{k}=\left(\begin{array}{cc}
\left(\rho_{k} c_{k}\right)^{2} & 0 \\
0 & 1
\end{array}\right)
$$

where $\theta \in \mathbb{R}^{+}, \mathbb{I}_{N-1}$ is the $(N-1) \times(N-1)$ identity matrix and for $k=1, . ., N, D_{k}$ is a $2 \times(N-1)$ matrix. The associated convection matrix is $\mathcal{Q}(\mathcal{U})=\mathcal{P}(\mathcal{U}) \mathscr{A}(\mathcal{U})$ with:

$$
\mathcal{Q}(\mathcal{U})=\left(\begin{array}{c|ccc}
\theta u_{1} \mathbb{I}_{N-1}+\sum_{k=1}^{N} D_{k}^{T} B_{k} & D_{1}^{T} C_{1} & \ldots & D_{N}^{T} C_{N} \\
\hline u_{1} D_{1}+P_{1} B_{1} & P_{1} C_{1} & & \\
\vdots & & \ddots & \\
u_{1} D_{N}+P_{N} B_{N} & & & P_{N} C_{N}
\end{array}\right) .
$$

We can easily see that the matrix $P_{k} C_{k}$ is symmetric for all $k=1, . ., N$. A necessary and sufficient condition for $\mathcal{Q}(\mathcal{U})$ to be symmetric is:

$$
\begin{aligned}
& \text { (i) }\left(C_{k}^{T}-u_{1} \mathbb{I}_{2}\right) D_{k}=P_{k} B_{k}, \quad \text { for all } k=1, . ., N \\
& \text { (ii) } \sum_{k=1}^{N} D_{k}^{T} B_{k} \text { is symmetric. }
\end{aligned}
$$

The matrix $C_{k}^{T}-u_{1} \mathbb{I}_{2}$ is a $2 \times 2$ matrix the determinant of which is $c_{k}^{2}\left(M_{k}^{2}-1\right)$ where $M_{k}=\left(u_{k}-u_{1}\right) / c_{k}$ is the relative Mach number of phase $k$. Hence, the matrices $C_{k}^{T}-u_{1} \mathbb{I}_{2}$ are invertible if and only if the non resonance condition (6) holds. Assuming (6), the matrix $D_{k}$ is therefore given by:

$$
D_{k}=\left(C_{k}^{T}-u_{1} \mathbb{I}_{2}\right)^{-1} P_{k} B_{k}
$$

An easy computation shows that the matrix $\left(C_{k}^{T}-u_{1} \mathbb{I}_{2}\right)^{-1} P_{k}$ is symmetric and we get that $D_{k}^{T} B_{k}=B_{k}^{T}\left(C_{k}^{T}-\right.$ $\left.u_{1} \mathbb{I}_{2}\right)^{-1} P_{k} B_{k}$ is also symmetric. Thus, condition $(6)$ is a necessary and sufficient condition for matrix $\mathcal{Q}(\mathcal{U})$ to be symmetric. The matrix $\mathcal{P}(\mathcal{U})$ is clearly symmetric. Therefore, it remains to prove that there exists $\theta>0$ 
such that $\mathcal{P}(\mathcal{U})$ is positive definite. Let $\mathbf{x}=\left(\mathbf{a}^{T}, \mathbf{b}_{1}^{T}, . ., \mathbf{b}_{N}^{T}\right)^{T} \in \mathbb{R}^{(3 N-1) \times 1} \backslash\{0\}$ with $\mathbf{a} \in \mathbb{R}^{(N-1) \times 1}$ and for $k=1, . ., N, \mathbf{b}_{k} \in \mathbb{R}^{2 \times 1}$. We have:

$$
\begin{aligned}
\mathbf{x}^{T} \mathcal{P}(\mathcal{U}) \mathbf{x} & =\theta \mathbf{a}^{T} \mathbf{a}+2 \mathbf{a}^{T} \sum_{k=1}^{N} D_{k}^{T} \mathbf{b}_{k}+\sum_{k=1}^{N} \mathbf{b}_{k}^{T} P_{k} \mathbf{b}_{k} \\
& \geq \theta|\mathbf{a}|^{2}-2|\mathbf{a}|\left|\sum_{k=1}^{N} D_{k}^{T} \mathbf{b}_{k}\right|+\sum_{k=1}^{N} \mathbf{b}_{k}^{T} P_{k} \mathbf{b}_{k}
\end{aligned}
$$

by the Cauchy-Schwarz inequality. The right hand side of this inequality is a polynomial of degree 2 in $|\mathbf{a}|$ and its second discriminant $\Delta^{\prime}$ is given by:

$$
\begin{aligned}
\Delta^{\prime} & =\left|\sum_{k=1}^{N} D_{k}^{T} \mathbf{b}_{k}\right|^{2}-\theta \sum_{k=1}^{N} \mathbf{b}_{k}^{T} P_{k} \mathbf{b}_{k} \\
& \leq N \sum_{k=1}^{N}\left|D_{k}^{T} \mathbf{b}_{k}\right|^{2}-\theta \sum_{k=1}^{N} \mathbf{b}_{k}^{T} P_{k} \mathbf{b}_{k} \\
& =N \sum_{k=1}^{N} \mathbf{b}_{k}^{T} D_{k} D_{k}^{T} \mathbf{b}_{k}-\theta \sum_{k=1}^{N} \mathbf{b}_{k}^{T} P_{k} \mathbf{b}_{k}
\end{aligned}
$$

again by the Cauchy-Schwarz inequality. Since $D_{k} D_{k}^{T}$ is symmetric and $P_{k}$ is symmetric positive definite, there exists an invertible $2 \times 2$ matrix $Q_{k}$ which simultaneously diagonalizes the two associated quadratic forms. More precisely, we have $Q_{k}^{T} P_{k} Q_{k}=\mathbb{I}_{2}$ and $Q_{k}^{T} D_{k} D_{k}^{T} Q_{k}=\delta_{k}$ where $\delta_{k}$ is a diagonal matrix. Defining $\overline{\mathbf{b}}_{k}=Q_{k}^{-1} \mathbf{b}_{k}$ we obtain:

$$
\Delta^{\prime} \leq \sum_{k=1}^{N} \overline{\mathbf{b}}_{k}^{T}\left(N \delta_{k}-\theta \mathbb{I}_{2}\right) \overline{\mathbf{b}}_{k} .
$$

Hence, choosing $\theta$ larger than the two eigenvalues of $N \delta_{k}$ for all $k=1, . ., N$ (observe that these eigenvalues only depend on $\mathcal{U}$ and not on the vector $\mathbf{x})$, we get that $\Delta^{\prime}<0$ and therefore $\mathbf{x}^{T} \mathcal{P}(\mathcal{U}) \mathbf{x}>0$ for all $\mathbf{x} \in \mathbb{R}^{(3 N-1) \times 1} \backslash\{0\}$.

\section{CONCLUSION}

We have proven the weak hyperbolicity, the existence of a convex mathematical entropy as well as the existence of a symmetric form. This last property is valid only far from resonance, i.e. as long as the model remain in its domain of hyperbolicity. These properties have been obtained for any admissible phasic equations of state (increasing phasic pressure laws). What is more, the proven properties can be extended to the two and three dimensional version of the model thanks to the frame invariance.

An important consequence of the symmetrisability and Kato's theorem on quasi-linear symmetric systems ( $[12]$ ) is that, far from resonance, there exists a unique local-in-time smooth solution to the Cauchy problem. The loss of regularity in finite time still holds, but with the additional restriction due to the non resonance condition (6).

It is worth mentioning that the techniques developed herein can be extended to similar systems modelling miscible compressible mixtures. We refer to 10 where symmetrisability results have been obtained for a threephase flow model where two of the present phases are miscible. 


\section{ACKNOWLEDGEMENTS}

The authors warmly thank Jean-Marc Hérard, who kindly discussed some of these results on a december $26^{\text {th }}$.

\section{REFERENCES}

[1] M.R. Baer and J.W. Nunziato. A two-phase mixture theory for the deflagration-to-detonation transition (DDT) in reactive granular materials. International Journal of Multiphase Flow, 12(6):861 - 889, 1986.

[2] J. B. Bdzil, R. Menikoff, S. F. Son, A. K. Kapila, and D. S. Stewart. Two-phase modeling of deflagration-to-detonation transition in granular materials: A critical examination of modeling issues. Physics of Fluids, 11(2):378-402, 1999.

[3] W. Bo, H. Jin, D. Kim, X. Liu, H. Lee, N. Pestieau, Y. Yu, J. Glimm, and J.W. Grove. Comparison and validation of multi phase closure models. Computers and Mathematics with Applications, 56(5):1291 - 1302, 2008.

[4] H. Boukili and J.-M. Hérard. Relaxation and simulation of a barotropic three-phase flow model. ESAIM: M2AN, 53(3):10311059, 2019.

[5] F. Coquel, J.-M. Hérard, K. Saleh, and N. Seguin. Two properties of two-velocity two-pressure models for two-phase flows. Commun. Math. Sci., 11, 2013.

[6] S. Gavrilyuk and R. Saurel. Mathematical and numerical modeling of two-phase compressible flows with micro-inertia. Journal of Computational Physics, 175(1):326 - 360, 2002.

[7] S. K. Godunov. An interesting class of quasilinear systems. Dokl. Acad. Nauk SSSR, 139:521-523, 1961.

[8] J.-M. Hérard. A three-phase flow model. Math. and Comp. Modelling, 45(5-6):732 - 755, 2007.

[9] J.-M. Hérard. A class of compressible multiphase flow models. Comptes Rendus Mathematique, 354(9):954 - 959, 2016.

[10] J.-M. Hérard and H. Mathis. A three-phase flow model with two miscible phases. ESAIM: M2AN, 53(4):1373-1389, 2019.

[11] D. Iampietro. Contribution to the simulation of low-velocity compressible two-phase flows with high pressure jumps using homogeneous and two-fluid approaches. Theses, Aix-Marseille Université, November 2018. https://tel.archives-ouvertes. fr/tel-01919156v1

[12] T. Kato. The Cauchy problem for quasi-linear symmetric hyperbolic systems. Archive for Rational Mechanics and Analysis, 58(3):181-205, Sep 1975.

[13] M. S. Mock. Systems of conservation laws of mixed type. J. Differential Equations, 37(1):70-88, 1980.

[14] S. Müller, M. Hantke, and P. Richter. Closure conditions for non-equilibrium multi-component models. Continuum Mechanics and Thermodynamics, 28(4):1157-1189, 2016. 